\section{Annual zoledronic acid infusion prevents bone loss in hypogonadal men}

Gonadotropin-releasing-hormone $(\mathrm{GnRH})$ agonists are routinely used to manage recurrent nonmetastatic prostate cancer, but the therapy results in hypogonadism, which has adverse consequences including osteoporosis. Zoledronic acid in 3-monthly doses has been shown to increase BMD in these patients; however, because high doses are associated with serious side effects, less frequent administration would be preferable. In a randomized, placebo-controlled study Michaelson et al. assessed the effect of a single, annual infusion of zoledronic acid on bone loss and BMD in men with nonmetastatic prostate cancer treated with $\mathrm{GnRH}$ agonists.

Eligible patients identified by bone scanning received either zoledronic acid $4 \mathrm{mg}(n=22)$ or placebo $(n=22)$ intravenously on day one of the study. All participants continued $\mathrm{GnRH}$ treatment for the duration of the study; 36 completed BMD testing at 12 months. In the placebo group BMD (mean \pm SE) decreased by $3.1 \% \pm 1.0 \%$ in the lumbar spine and by $1.9 \% \pm 0.7 \%$ in total hip from baseline. By contrast, in the zoledronic acid group BMD increased by $4.0 \% \pm 1.0$ in the lumbar spine and $0.7 \% \pm 0.5 \%$ in total hip. In addition, sustained decreases in levels of bone turnover markers were seen in the zoledronic acid group, in contrast to increases in the placebo group. No serious adverse effects were noted in either group.

The authors conclude that annual zoledronic acid therapy could be an effective therapy to prevent bone loss in hypogonadal men.

Original article Michaelson MD et al. (2007) Randomized controlled trial of annual zoledronic acid to prevent gonadotropin-releasing hormone agonist-induced bone loss in men with prostate cancer. J Clin Oncol 25: 1038-1042

\section{Molecular profiling of circulating tumor cells guides treatment selection}

Factors that contribute to prostate cancer growth and survival vary over time, which might mean that drug therapies lose effectiveness in a given individual. Assessment of the molecular profile of tumors through analysis of circulating tumor cells (CTCs) might help to target therapy. Shaffer and colleagues collected peripheral blood from patients with advanced prostate cancer and used immunomagnetic-capture technology to isolate and analyze CTCs.

Around $7.5 \mathrm{ml}$ of blood was collected from each of 63 patients with metastatic prostate cancer and from 17 controls without cancer at one US center. CTCs were captured with antibodies to the epithelial cell adhesion molecule and underwent immunofluorescent, Papanicolau staining and fluorescence in situ hybridization. To assess whether cell counts changed over time, additional samples were taken within $24 \mathrm{~h}$ of the first sample and at $72 \mathrm{~h}$ or at $96 \mathrm{~h}$.

The mean CTC count in patients with prostate cancer was 16 cells $/ 7.5 \mathrm{ml}$ blood (range 0847 cells). Subsequent CTC counts did not differ from those of the first samples. Cells expressed cytokeratin AE1/AE3 and $\alpha$-methyl CoA racemase. There was marked amplification of the androgen receptor locus in five patients with CTC counts of 50 or more. Four of these patients, and an additional two, showed signals of tetraploidy. No amplification of ERBB2 (HER2) was observed. The proportion of CTCs positive for EGFR ranged from $0 \%$ to $100 \%$ (median $56 \%$ ).

The authors hope that ongoing prospective studies will validate the markers identified, which might in turn improve clinical management of patients.

Original article Shaffer DR et al. (2007) Circulating tumor cell analysis in patients with progressive castration-resistant prostate cancer. Clin Cancer Res 13: 2023-2029 